'J Dubois, ' $\mathrm{G}$ Dehaene-Lambertz, ${ }^{2} \mathrm{JF}$ Mangin, ${ }^{3} \mathrm{D}$ Le Bihan, ${ }^{4} \mathrm{PS}$ Hüppi, ${ }^{5} \mathrm{~L}$ Hertz-Pannier. 'Cognitive Neuroimaging Unit, U 992, INSERM; ' LNAO; 'NeuroSpin, CEA, Gif-surYvette, France; ${ }^{4}$ Pediatrics Department, Geneva University Children's Hospital, Geneva, Switzerland; 5 LBIOM, CEA, Gif-sur-Yvette, France

Studying how the brain develops and becomes functional is a prerequisite to understand the complexity of developmental pathologies. Thanks to Magnetic Resonance Imaging (MRI), it is now possible to image the baby's brain non-invasively, and to provide subtle correlations between its anatomical development and the early acquisition of cognitive functions.

But imaging the immature brain implies several constraints at the levels of data acquisition and post-processing, because the baby may move, the examination length must then remain short, and because the size of cerebral structures and the image contrast are very different in comparison with the adult brain. Dedicated methodologies must then be applied to provide valid and instructive information on the development of cortical and white matter networks.

In this presentation, I will describe two recent MRI studies which evaluated the setting up of cortical morphology and connectivity under an original perspective. The first study has focused on the cortical folding patterns (formation of gyri and sulci), in preterm newborns from six to eight months of gestational age, by using T2-weighted conventional MRI. The second study has mapped the organization and maturation of major white matter fiber bundles (commissural, projection, association and limbic fascicles), in one- to four-months-old healthy infants, with diffusion tensor imaging (DTI) and tractography.

Both MRI studies have highlighted spatio-temporal differences in the maturation of brain regions, as well as early anatomical asymmetries between cerebral hemispheres. They have also emphasized the relationships between the structural development of the brain and the infant's psycho-motor acquisitions after birth.

\section{ARTERIAL SPIN LABELING MAGNETIC RESONANCE IMAGING TO ASSESS NEONATAL BRAIN PERFUSION}

doi:10.1136/archdischild-2012-302724.0194

${ }^{1} \mathrm{JB}$ De Vis, ${ }^{2} \mathrm{KJ}$ Kersbergen, ${ }^{1} \mathrm{ET}$ Petersen, ${ }^{1} \mathrm{~J}$ Hendrikse, ${ }^{2} \mathrm{~T}$ Alderliesten, ${ }^{2} \mathrm{~F}$ Groenendaal, ${ }^{2}$ LS de Vries, ${ }^{2}$ MJNL Benders. ${ }^{1}$ Radiology, UMC Utrecht; ${ }^{2}$ Neonatology, Wilhelmina Children's Hospital/UMC, Utrecht, The Netherlands

Background and aim Little is known about brain perfusion in neonates and its relation with brain development. The purpose of this study was to evaluate if Arterial Spin Labeling (ASL) Magnetic Resonance (MR) imaging can be used to evaluate the relation between brain perfusion and brain development.

Methods Pulsed ASL-images (Philips-3T) were acquired of 31 infants. Six infants were imaged at preterm age, 23 infants at term equivalent age (TEA) and 2 infants at 3 -months equivalent age $(3 \mathrm{~m})$. Serial MR imaging was performed in 4 infants. Total brain perfusion (TBP) was measured for each infant. Regions of interest (ROIs) were drawn within the occipital cortex (OC), the frontal cortex (FC) and one ROI covered the basal ganglia and thalamus (BGT). Perfusion values measured in the ROIs were expressed relative toTBP.

Results TBP increased from preterm age (7.1 $\mathrm{ml} / 100 \mathrm{~g}$. $\mathrm{min})$ to TEA $(12.6 \mathrm{ml} / 100 \mathrm{~g} \cdot \mathrm{min})$ to $3 \mathrm{~m}(30.2 \mathrm{ml} / 100 \mathrm{~g} \cdot \mathrm{min})$. A relative decrease in perfusion towards the BGT and a relative increase towards the $\mathrm{OC}$ and $\mathrm{FC}$ was measured with increasing postconceptional age [fig 1]. Serial ASL images of one infant scanned at preterm [Fig.2a] and TEA [Fig.2B] are shown.

Conclusions Arterial Spin Labeling MR images reflect the anatomical and functional maturation of the brain in neonates and are in agreement with previously obtained PET-images.

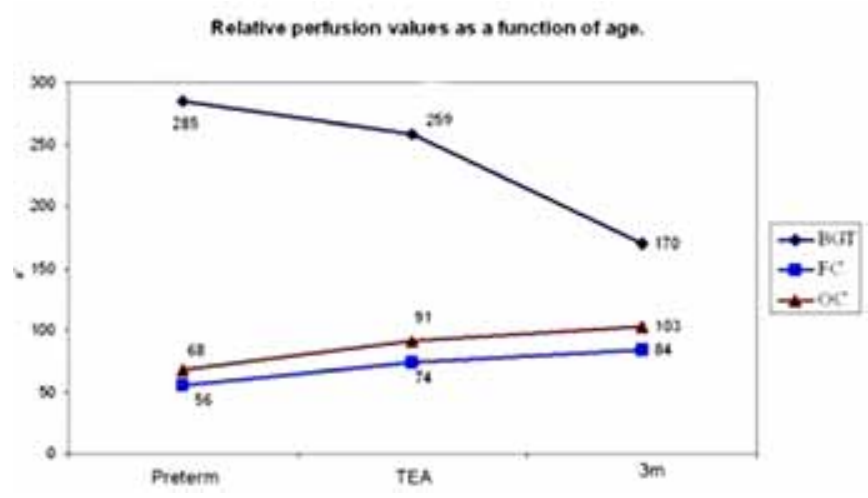

Abstract 194 Figure 1

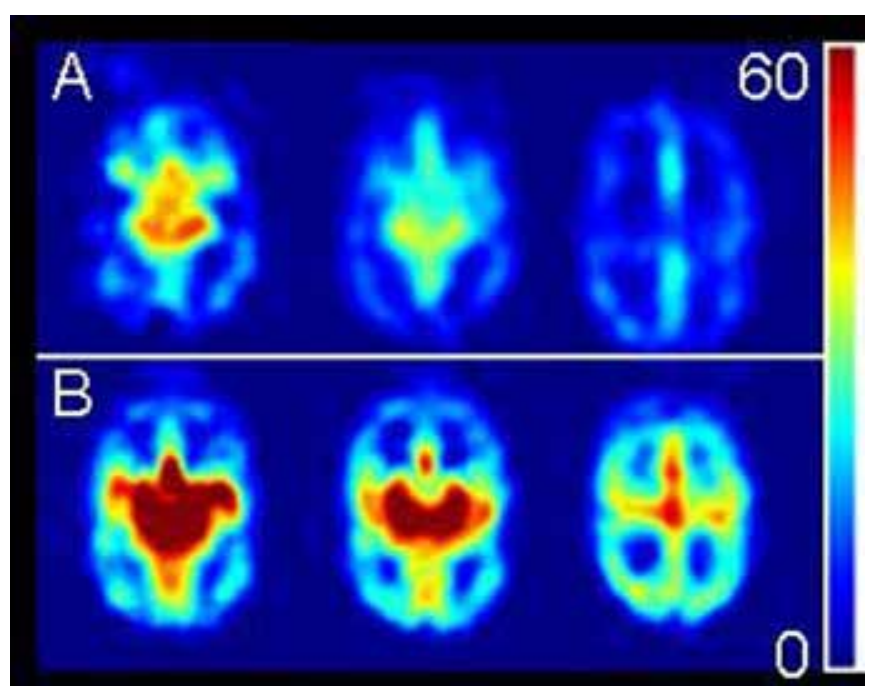

Abstract 194 Figure 2

\section{REGIONAL CHANGES OF CORTICAL THICKNESS AT 6 YEARS OF AGE IN PRETERM BORN CHILDREN}

doi:10.1136/archdischild-2012-302724.0195

'L Vasung, ${ }^{2} E$ Fischi Gomez, ${ }^{3} \mathrm{M}$ Monnier, ${ }^{4} \mathrm{AC}$ Evans, ${ }^{2} \mathrm{~F}$ Lazeyras, ${ }^{1} \mathrm{C}$ Borradori Tolsa 'PS Hüppi. 'Pediatrics, Service de Développement et Croissance, Geneva University Children's Hospital; ${ }^{2}$ Radiology, Center for Biomedical Imaging, Geneva University Hospital, Geneva; ${ }^{3}$ Pediatrics, Children's Hospital, Lausanne, Switzerland; ${ }^{4}$ ACE, Clinical Trials Imaging Lab, McConnell Brain Imaging Centre, Montreal Neurological Institute, McGill University, Montréal, OC, Canada

Background The growing incidence of prematurely born children and the improvement of survival rates have been associated with highly problematic long term neurodevelopmental outcomes. Brain structural alterations associated with these mainly cognitive difficulties most likely involve cortical organization. This study presents new ways of assessing structural organization of the cortex through thickness measurements.

Subjects/methods Preterm infants (N=42, GA $28.7 \pm 3.1 \mathrm{wks})$ were scanned at 6 years of age using the 3T MRI scanner. High-resolution 3D T1 MRI images were analyzed using MNI tools (http://www. bic.mni.mcgill.ca/ alan/lab.html). IUGR preterm subjects $(\mathrm{N}=14)$ were identified by abnormal antenatal Doppler measurements and mean birth weights below $10^{\text {th }}$ percentile. Cortical thickness was computed between extracted cortical surfaces and analyzed using the SurfStat tools (http://www.math.mcgill.ca/keith/surfstat/).

Results GA at birth within all subjects showed positive correlation with cortical thickness measurements (bilateral precuneus, right 
medial temporal gyrus, right cuneus, left inferior parietal lobule and left parieto-occipital arcus) at 6 years. The effect of preterm birth in the right junction of paracentral lobule and the precuneus and in the right transverse temporal gyrus shows statistically significant differences between groups $(p=0.001$, positively correlated with thickness at 6 years in the IUGR group and negatively correlated in the non-IUGR group).

Discussion/conclusion Our results indicate that the regional structural reorganization of cerebral cortex after preterm birth differs in IUGR and non-IUGR subjects. Preterm birth affects the higher order association areas with increased thickness or less thinning in IUGR than non-IUGR born children. These cortical changes might underlay the specific functional deficits observed in these children.

\section{IMPROVED DETECTION OF INTRACRANIAL HEMORRHAGE IN TERM AND PRETERM NEONATES USING SUSCEPTIBILITY WEIGHTED IMAGING}

doi:10.1136/archdischild-2012-302724.0196

${ }^{1} \mathrm{~S}$ Sirin, ${ }^{2 B}$ Huening, ${ }^{2} \mathrm{~A}$ Stein, 'SL Goericke, 'A Krasny, ${ }^{2} \mathrm{U}$ Felderhoff-Mueser, 'B Schweiger. 'Institute of Diagnostic and Interventional Radiology and Neuroradiology; ${ }^{2}$ Department of Neonatology, Division of Pediatrics I, University Hospital Essen, Essen, Germany

Background and aims Magnetic resonance imaging (MRI) at term age has been reported to be superior to cranial ultrasound (cUS) in detecting white matter abnormalities. The aim of this retrospective study was to compare sensitivity of MRI using SWI (susceptibility weighted imaging) and cUS in the detection of intracranial hemorrhage.

Methods 68 consecutive term and preterm neonates, who received 3 Tesla MRI of the brain with SWI (Magnetom Skyra, Siemens Healthcare, Erlangen, Germany) around term and serial cUS (Acuson sequoia 512, Siemens Healthcare) during neonatal care, were included in this study between 05/2011 and 02/2012. MRI was performed using a MR-compatible incubator with compatible head coil (LMT nomag, Luebeck, Germany) under sedation. MRI were analyzed by two radiologists independently. Inter-rater agreement was estimated by Cohen's kappa coefficient.

Results MRI and cUS were feasible in all 68 neonates (38 girls, 30 boys, mean gestational age at birth $31.9 \pm 4.5$ weeks (range 23.3-40.7 weeks). MR imaging was done at 40.3 \pm 3.0 weeks (range 30.7-55.7 weeks). Both radiologists independently identified (post-)hemorrhagic alterations in 20 of 68 infants by SWI (inter-rater agreement: $\mathrm{K}=1$ ). In 10 this was in agreement with cUS, but in 4 of them additional intraventricular and/or parenchymal hemorrhagic components were diagnosed by MRI. All patients with suspected intracranial hemorrhage by cUS were confirmed by SWI.

Conclusions We found improved detection of intracranial hemorrhage with high inter-rater agreement by MRI using SWI compared to cUS in term and preterm infants. All hemorrhages diagnosed by cUS could be confirmed by MRI

\section{SERIAL DIFFUSION TENSOR IMAGING DEMONSTRATES: WHITE MATTER MICROSTRUCTURE IN THE PRETERM PERIOD IS NOT RELATED TO GESTATION AT BIRTH}

doi:10.1136/archdischild-2012-302724.0197

1,2BM Huening, 'L Pazderova, 'G Ball, 'N Tusor, 'N Merchant, 'T Arichi, 'JM Allsop, ${ }^{2} \mathrm{U}$ Felderhoff-Müser, ${ }^{1} \mathrm{M}$ Rutherford, ${ }^{1} \mathrm{AD}$ Edwards, 'SJ Counsell. 'Centre for the Developing Brain, Department of Perinatal Imaging, Division of Imaging Sciences \& Bioengineering, King's College London, London, UK; ${ }^{2}$ Division of Pediatrics I, Neonatology, University Children's Hospital, Essen, Germany

Background and aims We have previously shown a dose-dependent effect of prematurity on white matter (wm) microstructure at term equivalent age (TEA). The aim of this study was to determine whether the degree of prematurity at birth is associated with FA values in the early neonatal period.

Methods Inclusion criteria: Preterm birth $<33$ weeks gestational age (GA), serial MRI and DTI, first scan $\leq 33$ weeks post-menstrual age (PMA), second at TEA.

We studied 52 preterm infants, with a median GA at birth of $27+{ }^{1}$ $\left(24^{+3}-32^{+6}\right)$ weeks. DTI data were analysed using tract based spatial statistics (TBSS). Voxel based statistics was performed to assess the correlation between GA at birth and FA, corrected for PMA at scan. Results Scan 1: $31\left(25^{+2}-33\right)$ weeks PMA.

There were no significant correlations between GA at birth and FA in any WM region.

Scan 2: $41^{+1}\left(38^{+6}-44^{+1}\right)$ weeks PMA.

GA at birth was significantly linearly correlated with FA values in the corpus callosum, internal and external capsule, optic radiation, cerebral peduncles, cingulum and inferior longitudinal fasciculus.

Conclusions These data suggest that diffuse wm injury is not an inevitable consequence of preterm birth, and imply there may be a window of opportunity between birth and term eqivalent age where intervention with appropriate treatments may ameliorate the adverse effects of prematurity on wm development.

\section{TRANSPLANTATION FOR PHT IN EARLY CHILDHOOD}

doi:10.1136/archdischild-2012-302724.0198

S Sweet. Pediatric Alllergy, Imunology and Pulmonary Medicine, Washington University, St. Louis, MO, USA

Compared to older children, where Cystic Fibrosis is the most common indication, pulmonary vascular disoders, either related to congenital heart disease or idiopathic pulmonary hypertension, make up $40-50 \%$ of the population of patients undergoing lung transplantation before 6 years of age. Lung transplantation of infants and young children is complicated by challenges in several domains including technical (i.e. airway complications), monitoring (transbronchial biopsies are more difficult to obtain and lung function testing requires sedation), developmental (in particular when oral motor development is delayed) and most importantly level of illness (more than $50 \%$ of infants and young children undergoing lung transplant require mechanical ventilation and/or extracorporeal support prior to transplant. Although improving in recent years, early outcomes lag behind older children and adults (70\% compared to $>80 \%$ ). However 3 and 5 year survival is comparable (65\% and $50 \%$ respectively), perhaps due to a lower incidence of acute and chronic rejection. Many children transplanted in infancy face developmental delays, most likely due to pretransplant insults. Nonetheless, excellent long term outcome is possible and should become increasingly likely with improvements in pretransplant support and management of posttransplant complicaitons.

\section{A HYBRID GENOME-KINOME HIGH-THROUGHPUT SCREEN REVEALS NOVEL MITOTIC TUMOR SUPPRESSOR SIGNALING AXIS}

doi:10.1136/archdischild-2012-302724.0199

'G Nalepa, ${ }^{2} \mathrm{~J}$ Barnholtz-Sloan, 'AS Lehmann, 'FC Yang, 'J Renbarger, 'DW Clapp. ${ }^{1}$ Indiana University School of Medicine, Indianapolis, IN; ${ }^{2}$ Case Western Reserve University Comprehensive Cancer Center, Cleveland, $\mathrm{OH}$, USA

Faithful cell division maintains genomic stability and prevents cancer. Our cells employ well-orchestrated signaling cascades to ensure meticulous segregation of the genome during mitosis. Failure of these checkpoint mechanisms jeopardizes genome integrity and promotes evolution of cancer cells. 